\title{
Stereotyping as a Major Barrier to Achievement of Interprofessional Education Competencies: A Narrative Literature Review
}

Christine Conroy

Midwestern University, cconro@midwestern.edu

Follow this and additional works at: https://nsuworks.nova.edu/ijahsp

Part of the Rehabilitation and Therapy Commons

\section{Recommended Citation}

Conroy C. Stereotyping as a Major Barrier to Achievement of Interprofessional Education Competencies: A Narrative Literature Review. The Internet Journal of Allied Health Sciences and Practice. 2019 Jan 01;17(3), Article 8.

This Manuscript is brought to you for free and open access by the College of Health Care Sciences at NSUWorks. It has been accepted for inclusion in Internet Journal of Allied Health Sciences and Practice by an authorized editor of NSUWorks. For more information, please contact nsuworks@nova.edu. 


\title{
Stereotyping as a Major Barrier to Achievement of Interprofessional Education Competencies: A Narrative Literature Review
}

\begin{abstract}
Purpose: A major advancement in interprofessional(IP) practice and education has been the introduction of the Interprofessional Education Collaborative (IPEC) core competencies. The purpose of this study is to explore stereotyping as a barrier to achievement of the IPEC competencies. Methods: There has been research into barriers to interprofessional collaboration and some barrier themes have occurred. But to this point, nothing has been studied on barriers to the use of the IPEC core competencies. This study aims to show barriers to achievement of the IPEC competencies through a narrative literature review. Articles were selected from three databases: CINAHL, Medline and ERIC were utilized in this review using the search terms "Interprofessional collaboration" and "Stereotyping". Results: In articles used to review barriers to interprofessional collaboration, an underlying theme of negative stereotyping about different professions appears to be in place. Themes include: Differences in history and culture, fears of diluted professional identity, differences in language and jargon, and concerns regarding clinical responsibility. Conclusion: A literature review of studies on implementation of interprofessional activities, with correlations to the IPEC core competencies as a framework suggest that stereotyping may be a major barrier to implementation and achievement of the IPEC competencies.
\end{abstract}

\section{Author Bio(s)}

Christine Conroy, PT, DPT, MHS, FNAP is an Associate Professor in the Physical Therapy Program at Midwestern University. She is also a Fellow of the National Academies of Practice. 


\title{
1IJAHSP \\ The Internet Journal of Allied Health Sciences and Practice \\ Dedicated to allied health professional practice and education
}

Vol. 17 No. 3 ISSN 1540-580X

\section{Stereotyping as a Major Barrier to Achievement of Interprofessional Education Competencies: A Narrative Literature Review}

\author{
Christine Conroy \\ Midwestern University \\ United States
}

\begin{abstract}
Purpose: A major advancement in interprofessional(IP) practice and education has been the introduction of the Interprofessional Education Collaborative (IPEC) core competencies. The purpose of this study is to explore stereotyping as a barrier to achievement of the IPEC competencies. Methods: There has been research on barriers to interprofessional collaboration, and some barrier themes have occurred. But to this point, nothing has been studied on barriers to the use of the IPEC core competencies. This study aims to show barriers to achievement of the IPEC competencies through a narrative literature review. Articles were selected from three databases: CINAHL, Medline, and ERIC. These databases were searched in this review using the search terms "interprofessional collaboration," and "stereotyping," and "IPEC competencies". Results: In articles used to review barriers to interprofessional collaboration, an underlying theme of negative stereotyping about different professions appears to be in place. Themes include differences in history and culture, fears of diluted professional identity, differences in language and jargon, and concerns regarding clinical responsibility. Conclusion: A literature review of studies on implementation of interprofessional activities, with correlations to the IPEC core competencies as a framework, suggest that stereotyping may be a major barrier to implementation and achievement of the IPEC competencies.
\end{abstract}

Keywords: interprofessional collaboration, stereotyping, IPEC competencies 


\section{BACKGROUND AND PURPOSE}

If those in interprofessional education are going to effectively address the issue of stereotyping, then a better understanding of what stereotypes are and where they come from needs to take place. Stereotypes have been an area of study in the social psychology arena for a long time. Hilton and vonHippel presented a thorough overview of the stereotyping literature in $1996 .{ }^{1}$ They ascertain that stereotypes are "beliefs about the characteristics, attributes, and behaviors of members of certain groups." ${ }^{1}$ The nature and purpose of the stereotypes may determine how and when the stereotypes are applied. The beliefs behind stereotypes come from two sources. First, there are mental representations of real differences between groups. So yes, stereotypes may represent accurate descriptions. In this case stereotyping allows for easy processing of information about others. However, the second source of stereotyping occurs when stereotypes are formed about groups independent of real group differences. This is the type of stereotyping that can become caustic to interprofessional endeavors.

How stereotypes are "represented" is a major issue in how stereotypes are formed. Different representational models lead to different ways of stereotypes being formed, maintained, and applied. ${ }^{1}$ Hilton presented 5 types of representation that lend to different types of formation and maintenance of stereotypes. These include prototype model, exemplar model, associative networks, schemas, and base rates. Formation may include self-fulfilling prophecies, non-conscious detection of covariance, illusionary correlation, and out-group homogeneity. Maintenance of stereotypes may occur through assimilation affects or attributional process. ${ }^{1}$ While the representation and formation of stereotypes is important, interprofessional education needs to focus on how to effectively resolve or even prevent these stereotypes from forming.

There have been several major milestones in the advancement of interprofessional practice and education over the past few years. Possibly the most significant of them is the introduction of the Interprofessional Education Collaborative (IPEC) core competencies for interprofessional education in the United States. ${ }^{2}$ Work on the competencies began in 2009 by the Institute of Medicine and World Health Organization and culminated with publication by the IPEC in May 2011 with 37 competencies in 4 domains2:

- Values/ethics for interprofessional practice

- Roles and responsibilities for collaborative practice

- Interprofessional communication

- Interprofessional teamwork and team based care

Each of these domains has several specific competencies for which the IPEC recently introduced strategies for implementation. There was a revision to these competencies by the IPEC leadership in 2016 when the "domains" became the core competencies with sub-competencies. ${ }^{3}$ The competencies were developed to provide educators with the key components for successful interprofessional practice. The competencies and strategies for interprofessional practice will lead the way for change in the healthcare system; however, the IPEC did not address barriers which may be present. While there is no current literature specific to the IPEC competencies, there has been research on barriers to interprofessional collaboration and education that include the following:4

- Differences in history and culture

- Historic interprofessional and interprofessional rivalries

- $\quad$ Differences in language and jargon

- Differences in schedules and professional routines

- Varying levels of preparation, qualifications and status

- Fears of diluted professional identity

- $\quad$ Differences in accountability, payment and rewards

- Concerns regarding clinical responsibility

Thought leaders in IPE and researchers have investigated these barriers, and stereotyping is implicated as a component within some of the barriers. Barr, for example, has stated that when learning about other healthcare professions, some attitudes about the other professions may come from ignorance, prejudice, or stereotypes, while other attitudes may be positive and mutually supportive. ${ }^{5}$ Similarly, a study from Pietroni looked at perceptions of students in social work, nursing, and medicine. ${ }^{6}$ Some of the demonstrated perceptions about the other professional groups were unexpectedly out of context, like social workers being "caring vegetarians" or nurses being "over-worked smokers." But the study also showed that the groups found solidarity in some of the congruent perceptions of each other. ${ }^{6}$ Ateah et al found that stereotypes existed among nursing students, but direct education on 
other health professions, and even more so, site immersion, could lessen these stereotypes. ${ }^{7}$ Stereotyping may be a major component of many barriers, but stereotyping may also be a person's or profession's way of creating organized representations of situations regardless of the authenticity of that representation. ${ }^{1}$

In addition, stereotyping may be a byproduct of a professional culture. In healthcare, values, beliefs, attitudes, customs, and behaviors have been developed reflecting historic factors, social class, and gender issues, as well as philosophical, societal, and economic models that change over time. ${ }^{8}$ The evolution of any healthcare profession includes a struggle to define its identity. Through these struggles, professions tend to build uni-professional silos that have common language, values, and problem-solving approaches. As professions work to develop their own cultures, they often form comparative views of other professions that are stereotypes of people who work within those professions. These stereotypes may be passed down with variation to novice practitioners who then carry the perceptions into their own practice. Through exclusionary closure, the profession limits the type of members and entrants into the profession so that the culture is passed down from one generation to the next with each neophyte practitioner being influenced by those who have come before them and carrying the stereotype forward. ${ }^{8}$ While such types of culturalization are important to professions developing their own identity, they can be counterproductive and create barriers to the collaborative efforts and competencies identified for IPE. Interprofessional Education strives to suspend stereotypes and help professionals and students better understand other professions, their roles, responsibilities, and boundaries. The current IPEC competencies do not directly address the issue of negative stereotypes, and based on the review of literature, there are no studies which have directly studied stereotyping as a barrier to implementation of the IPEC competencies. The purpose of this paper is to illustrate how stereotyping plays a significant role as a barrier to successful implementation of the IPEC competencies.

\section{Implications for Interprofessional Practice}

There are three primary implications of this study for interprofessional practice. First, if barriers to implementation to IP competencies are not addressed, IP activities may fail, and educators may see less value to the competencies. Second, if barriers, such as stereotyping can be identified, then perhaps activities can be implemented that address the barriers and make achievement of IPEC competencies more likely. Third, while these competencies were rigorously developed, research into the utilization and application of the competencies, as well as measurement of outcomes, still needs to occur.

\section{METHODS/LITERATURE REVIEW}

A review of literature was conducted using three databases (CINAHL, Medline, and ERIC). Search terms included "interprofessional collaboration" stereotyping," and "IPEC competencies." The following is a review of the literature found using these data bases and terms.

A stereotype is an over generalized representation of a group of people. Through stereotyping, we infer characteristics on a person we assume all group members possess. This inference may be positive, but often they are negative traits. These negative traits then entail a prejudice towards the group. Collaboration can be difficult if some members of a group hold stereotypes of others in the group. For communication to be productive, stereotyping must be avoided. One could infer that collaboration is primarily about communication, so stereotyping may be a barrier to promoting communication and collaboration. Interprofessional collaboration would also be at risk for break downs in communication via stereotyping. This is why one of the competency domains of the IPEC competencies addresses interprofessional communication. However, stereotyping may be a barrier to application of any of the domains.

The IPEC competencies fall under 4 major domains, each of which may be effected by stereotyping. The domain of Values/Ethics for Interprofessional Practice includes 10 specific competencies that address issues such as "...embracing cultural diversity and individual differences that characterize patients, populations, and the healthcare team" and "recognize the unique cultures, values, roles/responsibilities, and expertise of other health professions." ${ }^{2,3}$ In this domain, stereotyping may play a role in appreciating individual differences in patients or other professionals or valuing the expertise of another healthcare team member. In a study by Lewitt et al, medical students and biomedical science students were placed in an interprofessional lab course together. ${ }^{9}$ Preconceived ideas about the role of the biomedical science students by the medical students became a barrier to IP collaboration. The biomedical science students did not feel that an interprofessional course met their needs because "... it was completely wrong to give the doctors (medical students) a picture of us as laboratory technicians..." A comment from one of the medical students suggested that "the collaboration didn't really work. Mainly because the biomedical students couldn't bear to be called laboratory technicians..." 9 These preconceived ideas about the role of biomedical scientists can be seen as a stereotype which created conflict between the students. Working collaboratively in that type of environment would be difficult for any student or professional. 
One way to reduce this type of stereotyping is to directly address stereotypes about cultural differences and values early in the educational process. Reduced tension between and among students may increase collaboration in a course.

In a phenomenographical study of students in nursing, occupational therapy (OT), and social work (SW) in Sweden, Lidskog et al utilized an IPE experience and reflection as the basis to dispel stereotypes of each other's professions while on an interprofessional training ward for a three-week period. ${ }^{10}$ While not all preconceived ideas were dispelled, some attitudes were distinctly altered. Prior to the experience, OT and SW students viewed nurses as tending to "medical tasks." At the end of the experience, the OT and SW students had a greater appreciation for the nurse's co-coordinating role in the patient's health care. Nursing students perceived that the OT's role was more that of assisting other team members when indicated. At the end of the experience one student nurse explicitly addressed this conception stating, "I should say that I used to think it was more the nurse that called in the occupational therapist if help was needed - but now I can see that an occupational therapist has just as much contact with relatives and is just as involved in coordinating things." Nursing and OT students viewed the SW as an "outsider" prior to the IP experience. They felt that social work roles were outside the healthcare environment. At the end of the experience many of the nursing and OT students saw the role of the SW as advocating for the patient's wishes. ${ }^{10}$

The second domain presented by the IPEC competencies involves understanding roles/responsibilities for collaborative practice. 2,3 Holding inaccurate perceptions about the roles or responsibilities of another profession due to stereotyping can directly affect achievement of this competency. The interaction between the biomedical science students and the medical students from the Lewitt study showed that there was a skewed perception by the medical students of the role of the biomedical students as "lab technicians." In order to perform together in IPE, this type of stereotype would need to be addressed.

In a study by Sicher et al, several Eastern European countries embarked on an initiative to help curb the rampant increase seen in child abuse while these countries were emerging from government systems that were totalitarian in nature and did little with regard to child protection. ${ }^{11}$ One of the first challenges mentioned was the rigid hierarchies of the professionals. ${ }^{11}$ These types of rigid hierarchies can come from territorialism, but also from misconceptions about the education and roles of other professionals. ${ }^{8}$ Initial efforts to educate participants and break through stereotypes may improve the potential for success in IPP or IPE initiatives.

The third domain addresses the competencies involved in interprofessional communication. Most of the eight competencies listed under this domain are straightforward and deal with issues such as selecting effective communication styles. However, some are a bit unique and expand the view of "Interprofessional Communication" outside of these typical issues. For example, "Recognize how one's own uniqueness, including experience level, expertise, culture, power, and hierarchy within the health care team, contributes to effective communication, conflict resolution, and positive interprofessional working relationships - University of Toronto 2008."' One example of how stereotyping might effect this domain appears in an article by van Staa in $2000 .{ }^{12}$ In a palliative care unit in the Netherlands, an in-house interprofessional training included a weekly support group meeting which was recommended, but not mandatory. Participation was poor at first as facilitators and participants each had different views and expectations of the opportunity. Eventually, discussion revolved around problems with teamwork and collaboration, feelings of insecurity, and personal feelings of incompetence. ${ }^{12}$ Being able to freely discuss such frustration resulting from the perception of being un-empowered, level of education or expertise, and conflict may be a pathway to understanding each person's role in a collaborative team and to better understanding one's own perceptions of their role.

Recent trends in the provision of health care often involve multiple providers in order to provide unique specialized services. A study by Col et al looked at a diverse number of professionals utilizing shared decision making (SDM) within a primary care team. ${ }^{13}$ Understanding interprofessional sensitivities and acquiring relevant communication skills were two of the 5 areas targeted as being relevant for all stakeholders of the primary care team in order to effectively utilize SDM. Such sensitivities and unproductive communication may result from stereotyping towards certain professions. Another study by Conrad and Macrae titled "Turf, Team, and Town: a geriatric interprofessional education program" looked at how occupational therapy students can refine their professional parameters (turf) while still collaborating with other professionals (team) within a geriatric community (town). ${ }^{14}$ The scope of this study speaks to the challenge educators face in developing professional expertise, uniqueness, and cultural scope for students entering a health profession while trying to overcome the stereotypes that others might naturally attribute to these aspirations. Trying to develop a professional identity without actually creating comparisons that may turn to stereotypes is an area of interprofessional education that is just now being explored. Several studies are beginning to show that students may actually be entering professional studies with these stereotypes already imbedded, partially acquired from faculty who do not recognize their contributions. .15 
The fourth and final domain focuses on interprofessional teamwork and team-based care. While the third domain directly addressed communication among team members, it is the fourth domain that may be most affected by stereotyping. Communication is, of course, a vital skill and stereotypes may affect how we communicate within a team. However, the theme of teamwork moves beyond just communication skills and deals with the characteristics necessary for building and working within teams. If stereotypes exist, especially negative stereotypes, they may be harbored and not become apparent until there is stress on the team.

Teamwork has been largely defined as an important aspect for collaboration. ${ }^{16,17}$ While teams may differ in structure and how they operate, there is usually a clear relationship between the professions making up the team. So how individual professions might be preparing their students to work within interprofessional teams may play a role in successful teamwork. Miller et al proposed that uni-professional education may teach knowledge and skills for teamwork, but that they are not adequately involving students in actual teamwork where these skills can be practiced and refined. This was also true for multi-professional education programs. ${ }^{18}$ In a four-year action research study performed in Australia, 272 "substantial" interprofessional collaboration activities yielded no change in outcome scores when it came to the subscale of "Teamwork \& Collaboration." This was also true for the "professional Identity" subscale. ${ }^{19}$ The lack of sizable change in these two categories could be from a barrier like stereotyping.

Domain four speaks to specific competencies such as "Integrate the knowledge and experience of other professions...," "... constructively manage disagreements about values, roles, goals, and actions...," and "perform effectively on teams and in different team roles in a variety of settings." 2,3 If healthcare professionals harbor unrealistic stereotypes of other professions can they effectively acquire these competencies? An article by Reese and Sontag looked at the interprofessional role of hospice care. Despite inherent IP philosophy and treatment, barriers to full use of all disciplines on an interdisciplinary hospice team include lack of knowledge of the expertise of other professions, role blurring, and conflicts that arise from differences in professional values. ${ }^{20}$ Stereotyping can be the basis for some of these issues. In the Reese and Sontag article, they even point to client stereotyping as a potential barrier. ${ }^{20}$

According to Twaddle, the core concepts of teamwork include respect and dignity, information sharing, participation, and collaboration. ${ }^{21}$ In older models of teamwork, healthcare professionals considered themselves a team even if the team was a loosely structured group working in parallel or even duplicating services. Twaddle's newer model looks at the interdisciplinary team as "interdependent interaction." Effective functioning of such teams includes team composition/structure, organizational structure, and interpersonal relationships. Commitment to goals and environmental friendliness, as well as formal and informal information exchange influence these team characteristics. ${ }^{21}$ Stereotyping can harbor feelings of resentment and mistrust towards other professionals. Therefore, stereotypes may indirectly effect the development of well-functioning teams. Twaddle identifies ambiguity of team member roles and functions as one of the biggest barriers to teamwork: "... the result can be misunderstandings, resentment, and hurt."21 What if the misunderstanding and resentment are actually the impetus and not the result? In either direction, stereotyping can blur already ambitious roles even further, creating further "turf" issues.

\section{RESULTS}

The potential for stereotyping to act as a barrier to achieving the IPEC competencies appears to be present in all four domains. One productive method to introduce the competencies with minimal stereotyping may be to allow students to discuss their preconceived ideas and learn about a profession from the students in that profession. ${ }^{10,12}$ When collaborative efforts are attempted without first dispelling stereotypes, the results of the collaborative efforts seem to be less effective. 7,17

Additional methods may also be derived from the Contact Theory by George Allport.22-24 If groups can have an opportunity to communicate with each other, then they can have a better understanding of the other group and have new appreciation for the other group's point of view. However, the contact needs to occur under properly managed conditions. The conditions of contact that Allport felt were crucial to good outcomes included 1) equal status, 2) common goals, 3) intergroup cooperation, and 4) support of authorities, law or custom. ${ }^{22}$ Communication strategies with students or practitioners meeting to achieve common goals such as patient centered care plans or patient discharge plans may also be appropriate methods to bring about contact between different groups.

Several researchers have used "intergroup contact" as the theoretical framework for their studies in IPE initiatives. ${ }^{25-28}$ Bridges used intergroup contact; more specifically, the conditions of equal status and common goals, as the theoretical framework to explain positive attitude changes in interprofessional teams. ${ }^{27}$ Carpenter and Hewstone applied the "contact hypothesis" to final year social work and medical students and found that there were improvements in attitudes towards the other profession, and the 
participants reported an increased knowledge of the roles, attitudes, skills, and duties of the other profession. ${ }^{25}$ So there are known positive results from the application of intergroup contact as both a framework and intervention in IPE activities. As the study of IPE moves forward, there may be more ways to further explore the use of intergroup contact for decreasing stereotypes in interprofessional education.

\section{Recommendations for Future Research}

In each of the studies identified, contact was made by the different groups at the same time. The different professions were exposed to and learned about each other at the same time. What if a university is overcoming as many logistical barriers as possible and can only expose a few professional student groups to each other? Will this have an effect on how those students assimilate to other professional groups? Some areas which still need to be explored regarding stereotyping and IPE may include 1) Do student groups already participating in IPE activities have the same level of perceived stereotypes towards out-group professions as those not participating in IPE activities? 2) How does the introduction of a new professional group to an already existing IPE activity affect the attitudes and levels stereotyping of both the IP group and the out-group? 3) Does attitude change and improved knowledge occur at a different rate for the IP group compared to the newly introduced group? These questions are extensions of the work already performed using "intergroup contact" as a theoretical framework.

In terms of limitations of the included studies, one can see that there are similarities in the self-reported or qualitatively described use of stereotypes. Very few studies utilized a validated assessment tool of stereotyping such as Student Stereotypes Rating Questionnaire (SSRQ). Use of such a tool may have gleaned more accurate descriptions of the stereotypes perceived. Further research is needed to develop means of measuring impact of stereotyping to the IPEC competencies.

\section{CONCLUSION}

Given the proclivity for stereotyping as a barrier, there is a need for better understanding of stereotyping. Is it ever acceptable to hold stereotypes about other professions? Hall points out that stereotyping may be a simple byproduct in the development of a professional identity. ${ }^{8}$ How can medical educators use this information to both inform students about their own profession as well as promote collaborative efforts? Having a better understanding of stereotype formation, maintenance and tools for addressing stereotypes will further improve the efforts of interprofessional educators to write meaningful and effective IPE curriculum. And understanding the role stereotyping may play in achieving the IPEC competencies will further improve how educators utilize the competencies in their curriculum.

\section{References}

1. Hilton J, von Hipple W. Stereotypes. Annu Rev Psychol 1996;47:237-271. [PMID: 15012482]

2. IPEC. Core Competencies for Interprofessional Collaborative Practice: Report of an Expert Panel. http://www.aacom.org/InfoFor/educators/ipe/Documents/CCrpt05-10-11.pdf. Published 2011. Accessed May, 2017.

3. Interprofessional Education Collaborative. (2016). Core competencies for interprofessional collaborative practice: 2016 update. Washington, DC: Interprofessional Education Collaborative. https://www.ipecollaborative.org/resources.html. Accessed November 2018.

4. Headrick LA, Wilcock PM, Batalden PB. Interprofessional working and continuing medical education. BMJ (Clinical Research Ed). 1998;316(7133):771-774. [PMID: 9529419]

5. Barr H, et.all. Effective Interprofessional Education: Arguments, Assumption and Evidence.: Blackwell Publishing Ltd; 2005.

6. Pietroni P. Stereotypes or Archetypes? A Study of Perceptions amongst Health Care Students. Journal of Social Work Practice. 1991;5:61-69.

7. Ateah $\mathrm{CA}$, et al. Stereotyping as a barrier to collaboration: Does interprofessional education make a difference? Nursing Education Today. 2011;31(2):208-213.

8. Hall P. Interprofessional teamwork: professional cultures as barriers. Journal of Interprofessional Care. 2005;19:188-196. [PMID: 16096155]

9. Lewitt MS, Ehrenborg E, Scheja M, Brauner A. Stereotyping at the undergraduate level revealed during interprofessional learning between future doctors and biomedical scientists. Journal of Interprofessional Care. 2010;24(1):53-62. [PMID: 20001546] 
10. Lidskog M, Löfmark A, Ahlström G. Interprofessional education on a training ward for older people: students' conceptions of nurses, occupational therapists and social workers. Journal of Interprofessional Care. 2007;21(4):387-399. [PMID: 17654156]

11. Sicher $\mathrm{P}$, Lewis $\mathrm{O}$, Sargent $\mathrm{J}$, et al. Developing child abuse prevention, identification, and treatment systems in Eastern Europe. Journal Of The American Academy Of Child And Adolescent Psychiatry. 2000;39(5):660-667. [PMID: 10802985]

12. van Staa AL, Visser A, van der Zouwe N. Caring for caregivers: experiences and evaluation of interventions for a palliative care team. Patient Education And Counseling. 2000;41(1):93-105. [PMID: 10900371]

13. Col N, Bozzuto L, Kirkegaard P, et al. Interprofessional education about shared decision making for patients in primary care settings. Journal of Interprofessional Care. 2011;25(6):409-415. [PMID: 22011026]

14. Conrad SC, MacRae N. Turf, team, and town: A geriatric interprofessional education program. Work. 2012;41(3):285-292.

15. Mandy A, Milton C, Mandy P. Professional stereotyping and interprofessional education. Learning in Health \& Social Care. 2004;3(3):154-170.

16. Onyett S. Community mental health team working as a socially valued enterprise... Tensions in mental health policy?... Working together in adult community mental health services: an inter-professional dialogue. Journal of Mental Health. 1999;8(3):245-251.

17. Borrill C, Carletta J, Carter A, et al. The Effectiveness of Health Care Teams in the National Health Service. Birmingham: Aston Centre for Health Service Organisation Research, Aston Business School, Aston University. 2001:363.

18. Miller C, Freeman M, Ross N. Interprofessional Practice in Health and Social Care: Challenging the Shared Learning Agenda. Arnold, London2001.

19. Braithwaite J, Westbrook M, Nugus $\mathrm{P}$, et al. A four-year, systems-wide intervention promoting interprofessional collaboration. BMC Health Services Research. 2012;12:99-99. [PMID: 22520869]

20. Reese DJ, Sontag M. Successful interprofessional collaboration on the hospice team. Health \& Social Work. 2001;26(3):167-175. [PMID: 11531192]

21. Twaddle M. Teamwork--the new way. Health Progress. 2012;93(2):12-17. [PMID: 22423556]

22. Pettigrew T. Intergroup Contact Theory. In:1998:65-85.

23. Turner R. A Test of Extended Goup Contact Hypothesis. Journal of Personality and Social Psychology. 2008;94(4):843-860. [PMID: 18808263]

24. Dovidio J, A E, Hewstone M. Improving Intergroup Relations Through Direct, Extened and Other Forms of Indirect Contact. Group Processes \& Intrgrou Relations. 2011;14:147-160.

25. Carpenter J, Hewstone M. Shared Learning for Doctors and Social Workers: Evaluation of Programme. British Journal of Socail Work. 1996;26(2):239-257.

26. Carpenter J. Interprofessional Education for Medical and Nursing Studnets: Evaluation of Programme. Medical Education. 1996;29(2):265-272. [PMID: 8594389]

27. Brideges D, Tomkowiak J. Allport's Intergroup Contact Theory as a Theortical Base for Impacting Student Attitudes in Interprofessional Education. Journal of Allied Health. 2010;39(1):e29-33. [PMID: 20216998]

28. Barnes D, Carpenter J, Dickinson C. Interprofessional education for community mental health: attitudes to community care and professional stereotypes. Social Work Education. 2000;19(6):565-583. 